\section{COLOUR CHANGING STERILISATION POUCHES}

For a sterilisation pouch that combines a variety of features, choose PeelVue ${ }^{+}$from DUX Dental.

PeelVue $^{+}$sterilisation pouches feature both an internal and external processing indicator, which change colour after the sterilisation process is complete.

In addition, the PeelVue ${ }^{+}$Closure Validators offer a visual guide printed on the pouch to aid correct sealing of the pouch.

PeelVue $^{+}$sterilisation pouches are made from medical-grade, virgin non-recycled paper and leadfree inks and are easy to close and peel open, even while wearing gloves. Once opened and the transparent film removed, the paper side can function as a sterile tray liner.

With a useful colour coding to aid selection and available in 12 sizes, PeelVue ${ }^{+}$sterilisation pouches can be used in any steam auto/Chemiclave. Their easily removable clear film also provides prompt access to instruments.
Produced by DUX Dental, manufacturers and distributors of dental products for over 50 years, make PeelVue ${ }^{+}$sterilisation pouches your first choice.

For more information email info@dux-dental.com or visit www.dux-dental.com.

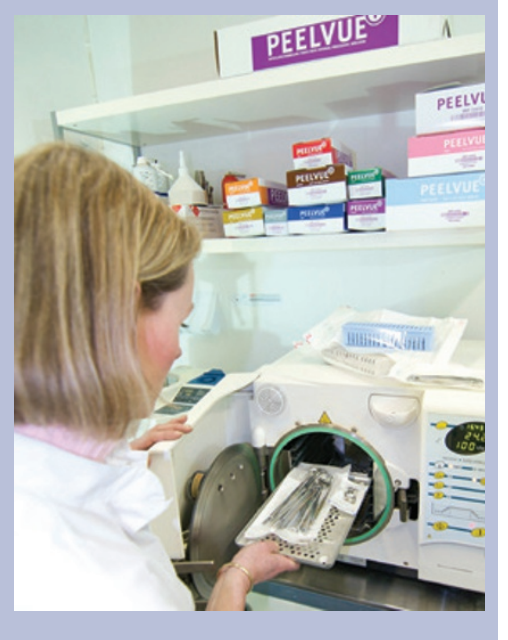

\section{CLEAN AND SHINING BRIGHT IN MINUTES}

\section{Alkazyme enzymatic is for the} thorough cleaning-pre-disinfection of all reusable, immersible dental instruments prior to autoclaving. It has a rapid cleaning action; when used in conjunction with an ultra-

sonic cleaner a minimum five minute contact time is all that is required to render used instruments thoroughly clean and shining bright. Alkazyme is also economical: just $5 \mathrm{~g}$ of Alkazyme with ordinary tap water makes 1 litre of enzymatic cleaning/disinfecting solution. Alkazyme is available from your usual dental sundry supplier in both $750 \mathrm{~g}$ loose powder and tubs of 100 easy dose water soluble sachets. www.alkazyme.com

\section{THE RESTORATIVE FOR ALL CLASSES OF CAVITY}

GrandioSO, the universal nano hybrid restorative for all classes of cavity, meets the highest demands for restorations in anterior and posterior regions. GrandioSO is suitable for class I to V restorations, reconstruction of traumatically injured anterior teeth, interlocking and splinting of loosened teeth, corrections of shape and shade to enhance aesthetic appearance, core build-up for crowns, and the fabrication of composite inlays.

GrandioSO has superb material properties and is the most toothlike material in the sum of its physical parameters. This composite allows for equally durable

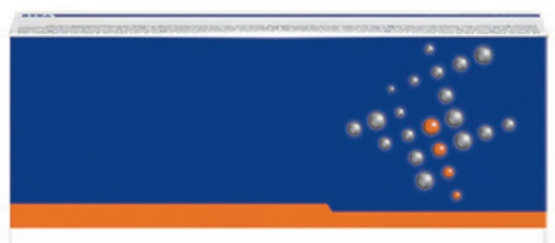

Grandieso

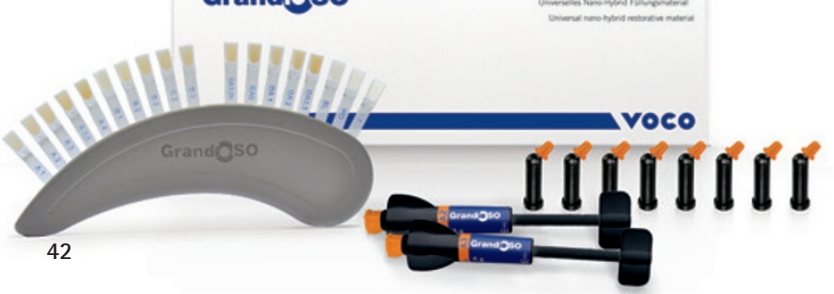

and aesthetic restorations, due to very high filler content and low shrinkage, high compressive and flexural strength, an E-modulus and thermal expansion behaviour similar to dentine, very high surface hardness, low abrasion as well as the optimal balance of translucence and opacity. Sixteen different shades cover the entire spectrum relevant to dentistry.

GrandioSO also convinces through its optimal handling properties. Due to its smooth consistency, this material is readily packable and possesses good sculptability without having the tendency to stick to the instrument. Grandioso is available in easy to use rotating syringes as well the particularly economical caps. VOCO is offering a GrandioSO Trial Pack as well.

www.voco.com

\section{QUALITY SERVICE A PRIORITY}

Providing a quality service to your patients is a priority for you as a dental professional, so shouldn't the service delivered to you be a priority for supply companies you work with?

Castellini has been creating truly innovative products for the dental industry for more than 70 years, and has become known for its exquisite craftsmanship and first class customer service.

Delivering complete support and maintenance services from centres located around the UK, the skilled team of engineers from Castellini are on hand to provide any advice or technical guidance you may need. They'll even offer training for the relevant members of your team.

So next time you need a new state-of-the-art dental unit, X-ray unit or specialist instruments, look no further than Castellini to receive customer service equal to the high standard of care your patients expect from you.

For more information on how to receive Castellini Technical Accreditation, call 08000933975 and speak to Castellini UK Ltd.

\section{EVERYTHING YOU NEED TO DISINFECT}

Eschmann offers the dental profession a complete solution for infection control with its Essential consumable range.

The collection features cleaning and disinfection products for use on surfaces, hands, instruments and special applications.

Other items in the range include detergent disinfectants for the treatment of dental instruments prior to sterilisation, sprays and wipes.

Delivering affordable and consistent market-leading quality - discover the Essential consumable range from Eschmann today.

For more information visit www.eschmann.co.uk or call 01903753322. 